\title{
Progress on the Reconstruction of a Dilution Refrigerator
}

\author{
Matthew Roberts*, Dustin Keller, Donald Crabb, and Blaine Norum \\ University of Virginia, USA \\ E-mail: mjr6mcevirginia.edu, dmk9m@virginia.edu, dgc3q@virginia.edu, \\ ben@virginia.edu
}

In this talk I will discuss the dilution refrigerator used in our lab for Polarized Target Physics. Originally constructed at CERN by T. Niinikoski et al. in the mid 1970's, modified by the Helmholtz Zentrum Geesthacht laboratory for neutron studies, and finally obtained by us at UVA for use in the HIGS collaboration. I will discuss the changes that have been made after we started using it. In November 2017, we discovered a leak in the still between two separate vacuum spaces and had no choice but to build a new dilution unit. I will lay out the progress we have made so far, what is currently happening, and the next steps that need to be taken.

The 18th International Workshop on Polarized Sources, Targets, and Polarimetry, PSTP2019

23-27 September, 2019

Knoxville, Tennessee

${ }^{*}$ Speaker. 


\section{Physics Motivations}

In 2006, researchers at LEGS sought to measure the Gerasimov-Drell-Hearn (GDH) Integrand for the neutron at photon energies below $400 \mathrm{MeV}$. However, since it is impossible to have a neutron target, they instead used the deuteron. At that time, the GDH Integrand for the proton was known and well-established, so they used that information to derive the neutron's GDH from measurements on the deuteron. One way they did this was to look at the ratios of these channels:

$$
\frac{p\left(\gamma, \pi^{+}\right)}{d\left(\gamma, \pi^{+}\right)} \rightarrow \frac{n\left(\gamma, \pi^{-}\right)}{d\left(\gamma, \pi^{-}\right)}
$$

By collecting data on the deuteron and using the information from the proton, researchers could find $n\left(\gamma, \pi^{-}\right)$. However, they found that the value they got had a non-negligible discrepancy with the theoretical value. After checking the data, they decided to look closer at the GDH Integrand. The GDH Integrand is a sum rule that relates experimentally measured cross-sections to the anomalous magnetic moment of the target particle. Both the target and the beam are polarized in this setup, with the two cases being parallel and anti-parallel. Parallel is when the beam and the target have the same polarization, and anti-parallel is when they have opposite polarizations. The GDH sum rule is given by:

$$
\int_{v_{T h}}^{\infty} \frac{\sigma_{P}-\sigma_{A}}{v} d v=\frac{4 S \pi^{2} \alpha \kappa^{2}}{m^{2}}[2]
$$

Where $v_{T h}$ is the threshold energy for the first inelastic channel (photodisintegration in the case of the deuteron), $\sigma_{P}$ is the parallel cross section, $\sigma_{A}$ is the anti-parallel cross section, $\mathrm{S}$ is the spin of the target, $\alpha$ is the fine structure constant, $\kappa$ is the anomalous magnetic moment, and $\mathrm{m}$ is the mass of the target particle. For the deuteron, one can expand the integral into three smaller integrals over three different ranges: from the photodisintegration threshold to pion threshold, from pion threshold to the maximum energy of the accelerator, and from the maximum energy to infinity. There are three possible explanations for this discrepancy: the GDH sum rule is wrong, there is something not accounted for in the high energy range (max energy to infinity), or there is something wrong with the extraction of the neutron integrand from the deuteron measurements that would be reflected in measurements on the deuteron below pion threshold. However, since the GDH sum rule is derived from well-established principles like quantum mechanics and Lorentz invariance, that possibility is highly unlikely. As for the second possibility, since the contribution to the anomalous magnetic moment goes like $\frac{1}{v}$ in the integral, it would have a small effect on the total sum, making it unlikely. Therefore, the most probable cause lies in the low energy regime.

That line of thinking is the basis of our collaboration at Triangle Universities Nuclear Laboratory (TUNL), and do most of our work at the Duke Free Electron Laser Lab (DFELL). In order to polarize our target deuterons, we have a dilution refrigerator, a superconducting magnet, and microwave system designed to help us achieve about 95 percent polarization. Downstream from the laser, we have our detector system, known as the Blowfish. It contains 88 liquid scintillator detectors set up at various angles to catch as many neutrons escaping as possible. We keep the dilution refrigerator at UVA in order to run practice cooldowns with it. The main purposes of these cooldowns are to make sure the fridge is in working order, optimize our ${ }^{3} \mathrm{He} /{ }^{4} \mathrm{He}$ ratio, and to help everyone involved master the procedure for the actual experimental run. 


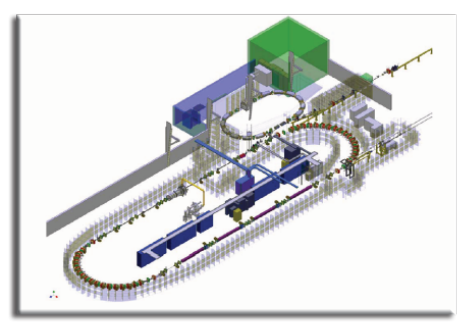

Figure 1: The accelerator at DFELL [4]

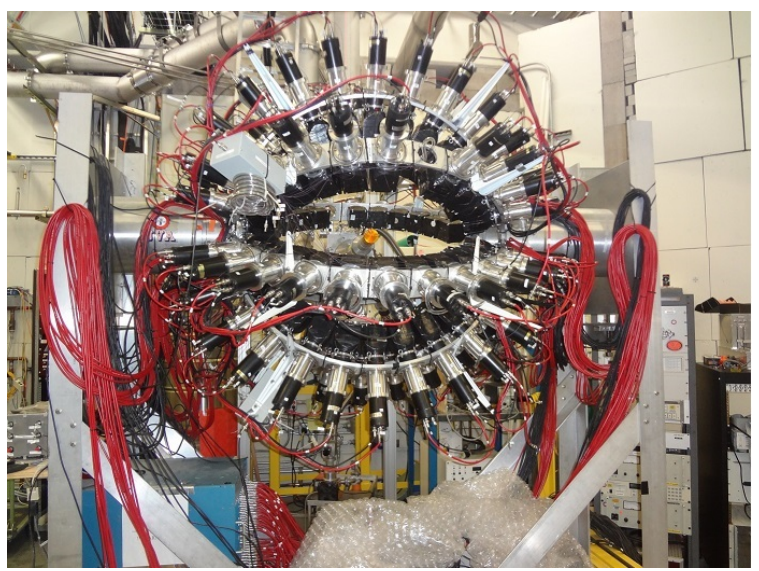

Figure 2: The Blowfish detector

The operating principle behind the dilution refrigerator lies in the ${ }^{3} \mathrm{He} /{ }^{4} \mathrm{He}$ ratio. When everything works, we take detailed notes of everything we do and record various temperature and pressure measurements over a long period of time, in order to replicate that process in the future. When things go wrong, we go over our notes to see if we did something wrong, or diagnose the problem and fix it before the next cooldown. Once we insert the liquid helium dewar into the fridge, the cooldown begins. Liquid ${ }^{4} \mathrm{He}$ is introduced into the fridge where it enters the evaporator. The evaporator pumps out the more energetic helium atoms, cooling the liquid helium to about one degree Kelvin. Circulated ${ }^{3} \mathrm{He}$ is cooled by this liquid helium bath, and then cooled further by the liquid inside the still. After leaving the still, the circulated He-3 enters the final heat exchanger, where it is cooled again by the helium mixture leaving the target chamber. When it does enter the target chamber, the almost pure ${ }^{3} \mathrm{He}$ sits on top of a "dilute" phase of ${ }^{3} \mathrm{He} /{ }^{4} \mathrm{He}$ that is mostly ${ }^{4} \mathrm{He}$. The ${ }^{3} \mathrm{He}$ atoms will penetrate through the barrier between these two phases, but it takes energy to do so. This leads to even more cooling, reaching temperatures in the low millikelvin range. The dilute phase then leaves the target chamber, cooling the incoming ${ }^{3} \mathrm{He}$ as it does until it reaches the still. The still is being constantly pumped on, removing the vapor that evaporates. This vapor is mostly ${ }^{3} \mathrm{He}$, and gets recirculated to repeat the process. While the dilution refrigerator is running, we would run the polarization process. We place the target chamber in the high magnetic field provided by the superconducting magnet, and bombard the target chamber with microwaves tuned to a specific frequency. Nucleons have a very low magnetic moment, and usually do not get 


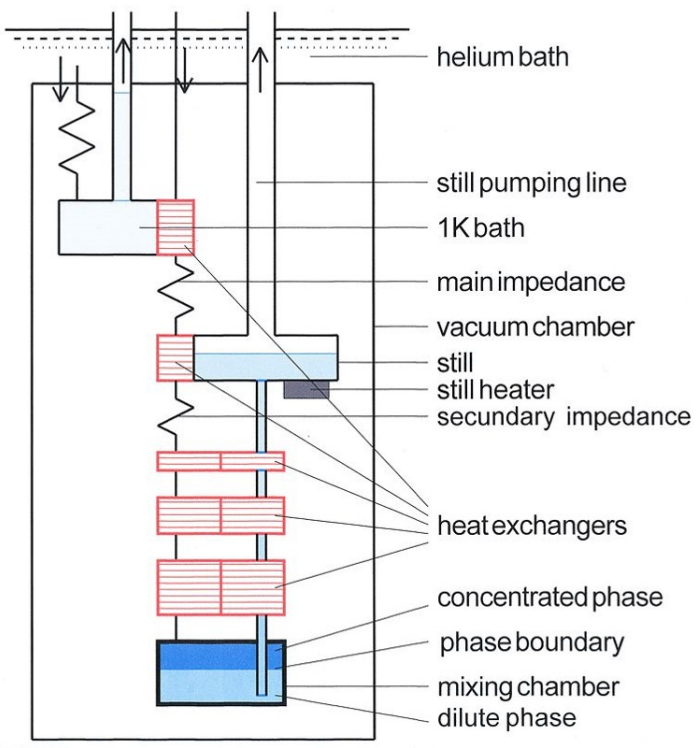

Figure 3: Schematic of a Basic Dilution Refrigerator [3]

polarized by the magnetic field alone. Instead, the microwaves "transfer" the polarization from the electrons to the nucleus in a process known as Dynamic Nuclear Polarization (DNP). We run this setup as a "Frozen Spin Target" meaning that we do not have to constantly bombard the target with microwaves to have it keep its polarization. When the target reaches a sufficient polarization, we turn off the microwaves and the polarizing magnet, move the target into the beam and start taking measurements.

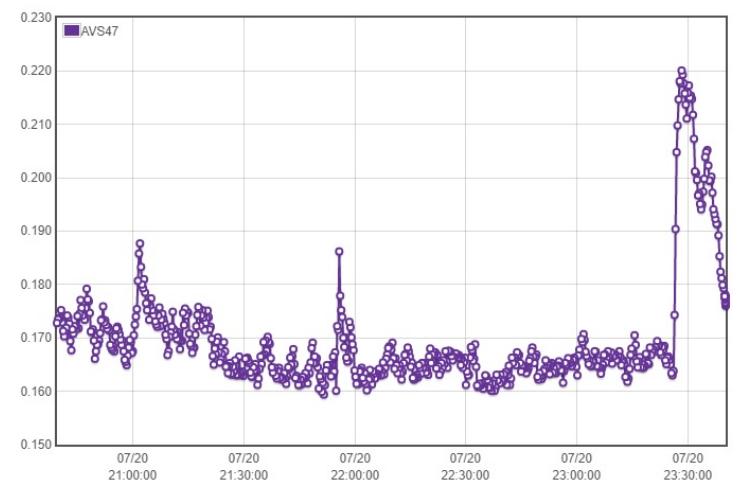

Figure 4: Target Chamber temperature from a successful cooldown

\section{History and Modifications}

Our dilution refrigerator was first constructed at CERN in the mid 1970's by Tapio Niinikoski for pion experiments. When those experiments ended, it was shipped to the Helmholtz-Zentrum Geesthacht facility in Germany for neutron experiments. However, since ${ }^{3} \mathrm{He}$ likes to absorb neutrons, the fridge was modified into a supercooled ${ }^{4} \mathrm{He}$ refrigerator to suit their needs. Next, we 
received the fridge after the Geesthacht group was done with it and began the process of removing their modifications to turn it back into a dilution refrigerator. Once that was finished, we began running cooldowns and preparing for our experiment. However, in November of 2017, we had an unsuccessful cooldown and had to stop it early. When we ran our diagnostics on the fridge, a leak in the still was discovered. Given that the still is at the heart of the refrigerator, we were left

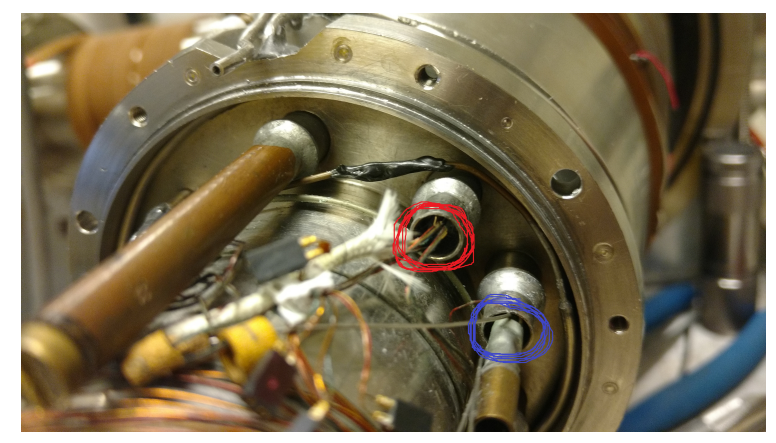

Figure 5: Picture of the fridge with the location of the leaks circled

with only one choice: remove the entire dilution unit from the fridge and build a new one. We slowly peeled off the layers and documented each step, taking pictures as we went. Once everything was disassembled, we gathered everyone and discussed how we would rebuild the unit. Our reconstruction had four basic ideas:

- It should replicate Niinikoski's original design, due to the amount of success he had with it.

- The new dilution unit should be easy to remove. The original was soldered on to the rest of the fridge and took over 2 hours for 3 people to remove. If this unit develops a leak in the future, we wanted to be able to get it out quickly.

- The new unit should not be held together with solder. One idea that was discussed was that the leak formed due to different thermal expansion coefficients of the solder and the stainless steel. Instead, this unit will be held together with indium seals, or welds.

- There should be only one still bypass tube. The old unit had two of these. The bypass tubes allowed wires to get past the still without coming into contact with the $1 \mathrm{~K}$ liquid and heating it up.

We began taking measurements of the old parts as well as the fridge, and began making SolidWorks models for the new pieces.

\section{Progress Made}

The first hurdle we had to clear in our design was how to make a seal between the new dilution unit and the fridge. Thus, the first part that was designed and made was an "Indium Adapter Ring". This ring was made to be welded on to the fridge, and form an indium seal with the dilution unit. Indium seals keep everything vacuum tight, but it also adheres to the ease of removal idea. It's a very simple design, with a groove for the indium and screw holes for the mating part of the dilution 
unit. A cap was made for this part, with the sole purpose of testing the leak tightness of this part after it was welded on. Once the cap was attached, we used a leak checker on it, both at room temperature and at $77 \mathrm{~K}$. When we found that it was leak tight in both scenarios, we moved on to the next piece. The next part that we had made was the still. We had it made in five separate parts:

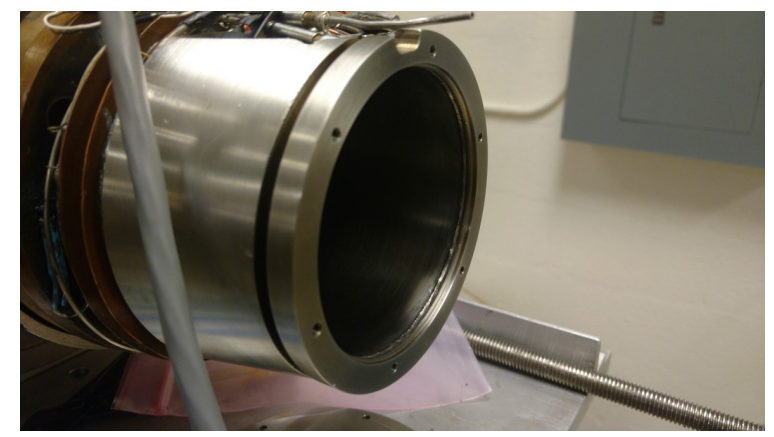

Figure 6: The Indium Adapter Ring on the fridge

The body, the front face, the back face, the ${ }^{3} \mathrm{He}$ pumpout, and the still bypass tube. The still itself is just a container for the Helium mixture that left the target chamber, with the pumpout used as the escape for the evaporated ${ }^{3} \mathrm{He}$ vapor to be recirculated. None of the parts have been welded together yet because we want all of the parts in our hands to check fits and clearances before they're fused together. Next, we have the central tube. This tube will eventually we welded to the back of

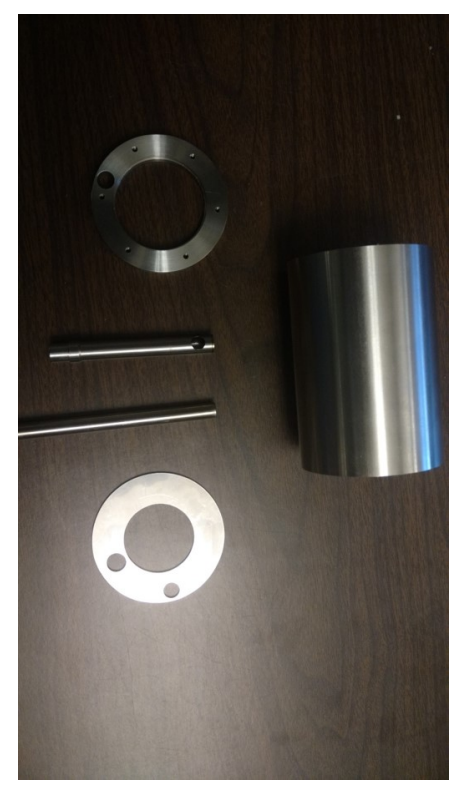

Figure 7: The unassembled still

the Vacuum Layer Can and the still, with the idea of keeping those two vacuum spaces separate. The final heat exchanger will be wrapped around this central tube. The final heat exchanger will be a cupronickel tube surrounded by sintered copper, with the incoming ${ }^{3} \mathrm{He}$ traveling down the cupronickel tube and the outgoing mixture traveling coaxially through the sintered copper. Since the outgoing mixture is mostly ${ }^{4} \mathrm{He}$, it will be superfluid, allowing it to travel along the sides of the 
central tube. In order to make sure it follows along the path of the heat exchanger, a nylon rope will be wrapped around the central tube as well, creating a channel for the superfluid to follow. One change we made to this central tube was the addition of a baby bottle cap shaped end on it. This end will have a very thin layer of stainless steel at the tip. This end has a two-fold purpose: To ensure the target beads are pushed in enough, and as a sort of window for the photons from the beam to travel through. Next, we made our Vacuum Layer Can (VLC). Its main purpose is

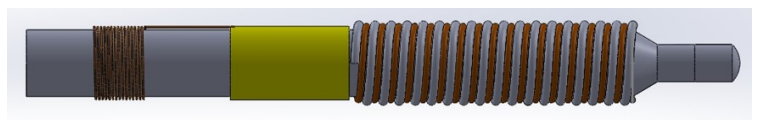

Figure 8: Solidworks drawing of the new central tube, with the heat exchanger and rope

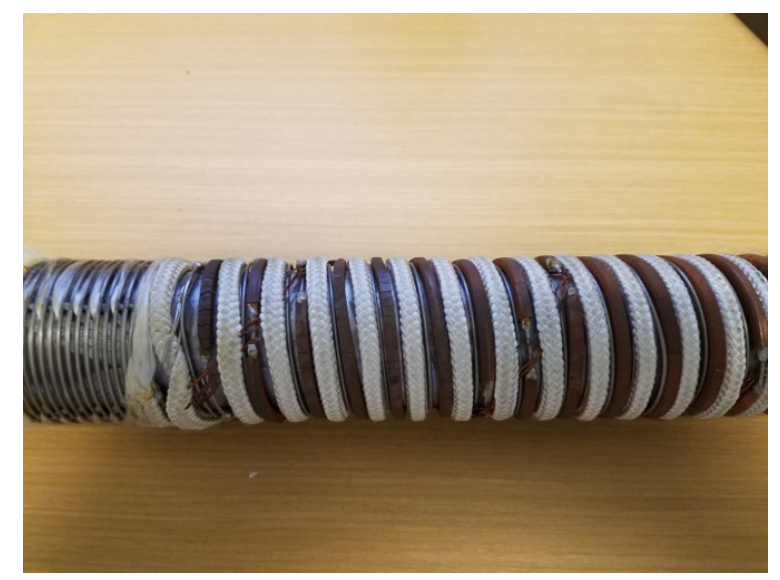

Figure 9: Old Central Tube with heat exchanger and rope channel

to provide a layer of insulation for the still. The biggest change we made to the new one is the addition of a double indium seal to the lip. Like the old one, it has an indium seal for mating with the Inner Vacuum Chamber (IVC) on the front side, and a seal on the backside for the adapter ring. It has two sets of screw holes for these seals, with the screw holes for the adapter ring being sunken, so that the heads of the screws do not interfere with the IVC. Both indium seals have been

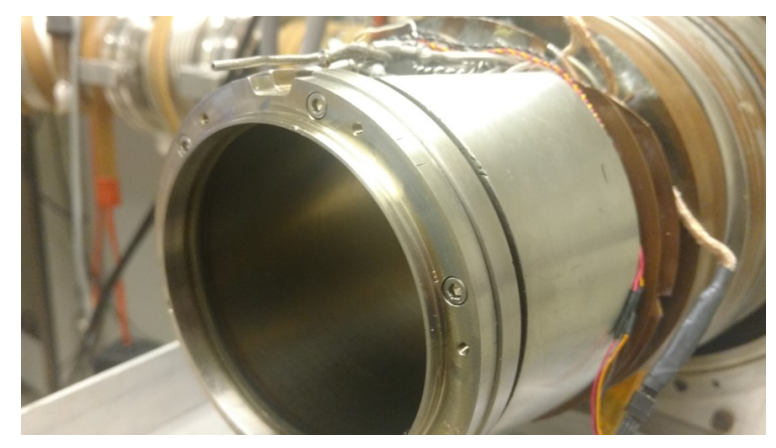

Figure 10: The VLC in the fridge

tested at room temperature and at $77 \mathrm{~K}$, with no leaks found. We also ran an evaporation cooldown 
(a cooldown without dilution) with these seals, which was a success. We were able to maintain $1 \mathrm{~K}$ in the evaporator over a time period of several hours without supervision or adjustments. The final part that has been made is referred to as the "sleeve". It forms an indium seal with the still and the target chamber and slides on the central tube. On the old setup, this part was welded to the still, preventing access to the heat exchanger inside. The inner diameter of this part was chosen carefully in order to make sure that it compressed the rope on to the central tube, in order to ensure that the superfluid helium can not climb over the rope. It also forms the other half of the ${ }^{3} \mathrm{He}$ vacuum space, (the first half was provided by the welds on the central tube) ensuring that the ${ }^{3} \mathrm{He}$ is recirculated and not pumped out. At the time that this is being written, (February 2020) we have

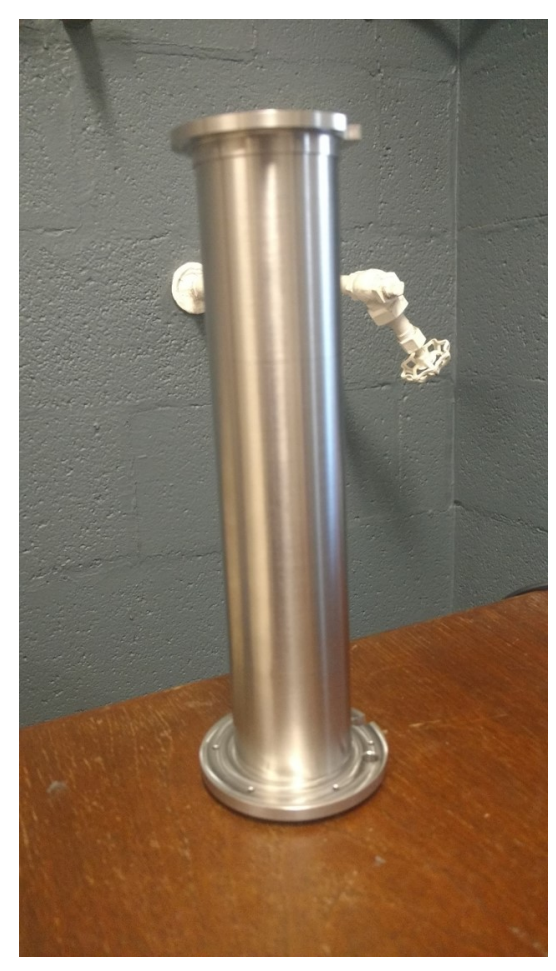

Figure 11: The Sleeve

obtained the waveguide, and are currently in the process of reconstructing the bellows that will go around it. We've redesigned them, and have most of the parts ready. We have also received a cryogenic feedthrough (courtesy of Christopher Keith at Jefferson Lab) and will soon attach the wires for the sensors to them, and then weld the feedthrough to our bellows.

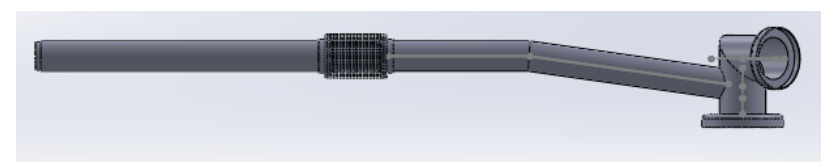

Figure 12: The final design of the new bellows

\section{Next Steps}

The next step for us is to weld all of the parts of the bellows together with the waveguide 
inside. Once the wires are attached to the feedthrough, that will also be welded to the bellows. We will then attach four temperature sensors to the inside of the still with epoxy, and then connect them to the feedthrough. The main purpose of these sensors is to monitor the liquid inside the still during a cooldown. Two will be placed low, and the other two placed high, letting us know when there is too much or too little liquid inside. We have two at each level in case a sensor breaks in the future. Once we have all of our electrical connections secured, we will weld all of our parts together. After we insert the completed dilution unit, we will run our first cooldown with it almost immediately. This cooldown will occur sometime this year, and once we confirm that we can achieve dilution with it, we will begin planning our experimental run.

\section{References}

[1] T.O.Niinikoski and F.Udo, "Frozen Spin" Polarized Target, Nuclear Instruments and Methods 134 (1976) 219.

[2] S. Hoblit, et al., Measurements of $\vec{H} \vec{D}(\vec{\gamma}, \pi)$ and Implications for the Convergence of the Gerasimov-Drell-Hearn Integral, LEGS-Spin Collaboration, Physical Review Letters 102 (2009) 172002.

[3] Dilution Refrigerator, https://en.wikipedia.org/wiki/Dilution_refrigerator

[4] , Triangle Universities National Laboratory www.tunl.duke.edu 